\title{
PRIMARY PANCREATIC LYMPHOMA WITH HERPES ZOSTER ASSOCIATED ACUTE PANCREATITIS: A CASE REPORT AND REVIEW OF LITERATURE.
}

\section{Vikram \\ Chaturvedi* \\ Chaturvedi \\ Sharmistha \\ Debnath}

\author{
Medical College \& Hospital, Kolkata, India. *Corresponding Author
}

ESIC Hospital and PGIMSR, Joka, Kolkata, India.

\section{Ābhishek Mishra}

Medical College \& Hospital, Kolkata, India.

Medical College \& Hospital, Kolkata, India.

ABSTRACT Primary pancreatic lymphoma (PPL) is an extremely rare disease, with few cases reported in literature. Clinical manifestations are usually nonspecific and mimic other pancreatic diseases. Owing to paucity of data and experience of PPL, clinico-pathological features, differential diagnosis, optimal treatment and overall outcomes are not well known.

Varicella zoster virus (VZV) is known to cause varicella in children and herpes zoster in adults. Acute pancreatitis caused by $\mathrm{VZV}$ is a rare and serious complication, with immunocompromised individuals being primarily affected.

We report a case of VZV associated acute pancreatitis in a patient who was diagnosed and treated for primary pancreatic lymphoma, thus rendering her immunocompromised and susceptible to developing infectious pancreatitis. A 56 year old female patient presented with recurrent vomiting, upper abdominal pain and hyperaesthesia in the right upper quadrant of 2 days duration. Following admission her right sided abdominal pain worsened (stabbing pain) and a diagnosis of acute pancreatitis was confirmed based on elevated serum amylase levels and CT scan findings of pancreatic enlargement. The patient was managed conservatively with intravenous fluids, antibiotics, analgesics and topical acyclovir ointment. The patient continues to be asymptomatic after 5 years of follow up. Though extremely rare, a diagnosis of herpes zoster pancreatitis should be considered in such patients, and prompt treatment initiated to prevent further deterioration and to minimize mortality.

Core Tip: Primary pancreatic lymphoma is in itself a very rare disease, and our patient having presented with herpes zoster associated acute pancreatitis in a background of PPL makes this case even rarer. This is probably the first case report of herpes zoster associated acute pancreatitis in an immunocompromised patient due to primary pancreatic lymphoma.

KEYWORDS : Primary pancreatic lymphoma, diffuse large B cell lymphoma, varicella zoster virus, acute pancreatitis.

\section{INTRODUCTION:}

Primary pancreatic lymphoma (PPL) is an extremely rare disease, accounting for less than $0.5 \%$ of all malignant pancreatic tumours (1-2), and is difficult to diagnose with accuracy even with various imaging modalities.

Clinically the presentation of PPL arising from pancreatic head region and pancreatic adenocarcinoma are similar and difficult to distinguish radiologically. However, prognostically both are quite different. PPL carries a much better prognosis, and hence proper histological diagnosis is of paramount importance as most pancreatic head masses end up with needless resection. Management of PPL requires chemoth erapy and radiotherapy (3).

This 56 year old female patient had initially presented with jaundice, upper abdominal pain and severe nausea with occasional vomiting. Clinically, apart from icterus, there was no organomegaly or peripheral lymphadenopathy. Liver function tests revealed raised total and direct component of bilirubin, increased transaminase and alkaline phosphatase levels. Serum amylase was marginally raised while lipase, LDH, CEA \& CA19-9 levels were in the normal range. CT scan of abdomen showed a pancreatic head mass $3.0 \times 3.2 \mathrm{~cm}$, with dilated common duct $(9 \mathrm{~mm})$ but normal MPD. There was no hepatomegaly, splenomegaly or retroperitoneal nodes on CT scan. CT guided biopsy revealed diffuse large B-cell lymphoma. Immunohistochemical staining showed cells to be positive for CD-20 and CD-79a (B-cell markers), and negative for CD-3, CD-10 and pancytokeratin. Based on pathological and IHC findings, a diagnosis of primary pancreatic lymphoma was made and the patient received 6 cycles of rituximab with cyclophosphamide, doxorubicin, vincristine and prednisolone (R-CHOP regimen). Following completion of 6 cycles of chemotherapy, a repeat contrast CT scan abdomen and thorax showed complete disappearance of the pancreatic head mass.

Around 4 months after completion of her last cycle of chemotherapy, she was admitted with recurrent vomiting, upper abdominal pain and hyperaesthesia in the right upper quadrant. Following admission the right upper abdominal pain worsened (stabbing pain) along with an increase in hyperaesthesia. Serum amylase levels were increased and contrast CT scan of abdomen showed pancreatic enlargement (Balthazar grade-B). This was followed by the development of a sheet of extremely painful vesicular rash along the right thoracodorsal area. In view of physical, biochemical and radiological findings, a diagnosis of herpes zoster associated acute pancreatitis was made and the patient was treated in the line of pancreatitis with gastric decompression, oxygen inhalation, intravenous fluids and antibiotics, analgesics and topical acyclovir ointment. After amylase and CRP levels normalized, the patient was started on enteral feeds and normal diet was allowed thereafter. The rashes too took around 10-12 days to heal. She was discharged from the hospital on the $15^{\text {th }}$ day. The patient has since then been on regular follow up for the last five years and is presently asymptomatic. 
DISCUSSION:

Primary pancreatic lymphoma (PPL) is an extremely rare disease, accounting for less than $0.5 \%$ of all malignant pancreatic tumours. The most common lymphoma variant in pancreas is diffuse large B-cell lymphoma (DLBCL), accounting for around $80 \%$ of all PPL. Symptoms of upper abdominal pain, jaundice, vomiting, acute pancreatitis or bowel obstruction are often non specific and overlap those of pancreatic adenocarcinoma. Typical B symptoms of night sweats, fever, chills are present in only $2-5 \%$ patients. The criteria for diagnosis of PPL as laid down by Dawson et al(4), include a dominant mass in pancreas, absence of superficial and mediastinal adenopathy on chest imaging, normal leucocyte count and the absence of hepatic or splenic involvement. Most common location of PPL is the head region(5). Biochemical and serum tumour marker, CA19-9 are rarely raised in primary pancreatic lymphoma. It has been observed that a high value of LDH and B2 microglobulin confers poor prognosis for these patients.

Various imaging modalities have been used for the evaluation of pancreatic masses. PPL may either present with radiological features similar to pancreatitis or a well circumscribed tumour. Features in favour of PPL are localized head of pancreas mass with normal MPD, no enlarged abdominal nodes and no hepatic or splenic involvement.

Since clinically, biochemically and radiologically it is difficult to diagnose PPL, biopsy becomes important to seal the diagnosis. EUS guided FNAC or CT guided biopsy are options for obtaining tissue for evaluation(6). Occasionally laparoscopy or laparotomy may be needed to get tissue from the mass or node for diagnosis.

Treatment of PPL consists of surgery if all investigations prove noncontributory, chemotherapy, radiotherapy or a combination of them. Chemotherapy consists of CHOP or R$\mathrm{CHOP}$ regimen if $\mathrm{CD}-20$ positive. Majority of patients remain disease free after receiving only chemotherapy(7).

Only a few cases of herpes zoster associated acute pancre atitis have been reported, most of them in immunocom promis ed patients, such as those in ICU, AIDS patients, those on long term immunosuppression eg. Stem cell (11-12)/ renal/ liver transplant patients. The exact mechanism of pancreatitis by herpes zoster remains unknown. The virus may remain latent in the posterior sensory nerve roots $(8,10)$, that contain fibres of skin, abdominal viscera including pancreas. VZV might injure the pancreatic acinar cell membrane resulting in leakage of intracellular enzymes, thus leading to pancreatitis. As the patient was a known case of primary pancreatic lymphoma who had received 6 cycles of chemotherapy and was possibly immunocompromised, along with the typical rashes of herpes zoster and biochemical and radiological evidence of acute pancreatitis, we were able to come to a diagnosis of VZV associated pancreatitis, as other common causes like gallstones, alcohol, infections(9) had been excluded. Management of this condition is similar to those caused by other etiologies, involving supportive treatment, fluid resuscitation, analgesics, enteral or parenteral nutrition.

\section{CONCLUSION:}

We have presented an extremely rare case of herpes zoster associated acute pancreatitis in a known patient of primary pancreatic lymphoma. It is important to distinguish between PPL and adenocarcinoma as PPL has a better prognosis and the treatment differs widely from that of carcinoma. Hence a histological diagnosis should be sought by way of image guided biopsy. Combined therapy may be the optimal treatment, but needs further evaluation.

Although rare, herpes zoster may be considered as a differe ntial diagnosis of acute pancreatitis in immunocompr omised individuals, particularly when the location and quality of pain change during the course of the illness. Prompt treatment is of paramount importance to prevent further deterioration and to minimize mortality.

\section{Term Explanation:}

Herpes zoster refers to the reactivation of a latent varicella zoster virus in the presence of a favourable environment to replicate and cause infection.

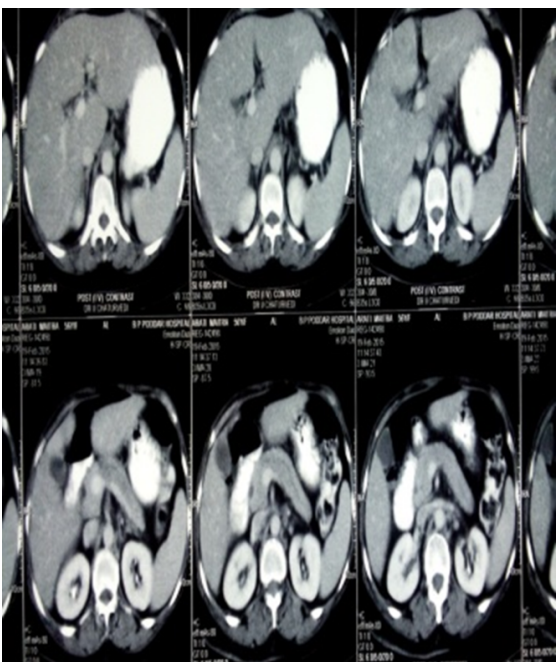

Figure Showing Diffuse Pancreatic Enlargement.

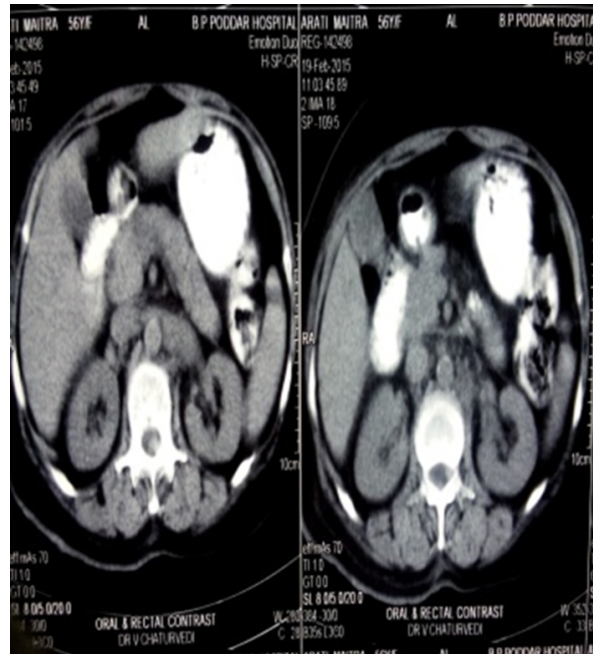

Figure Showing Pancreatic Head Enlargement Due To Pancreatitis

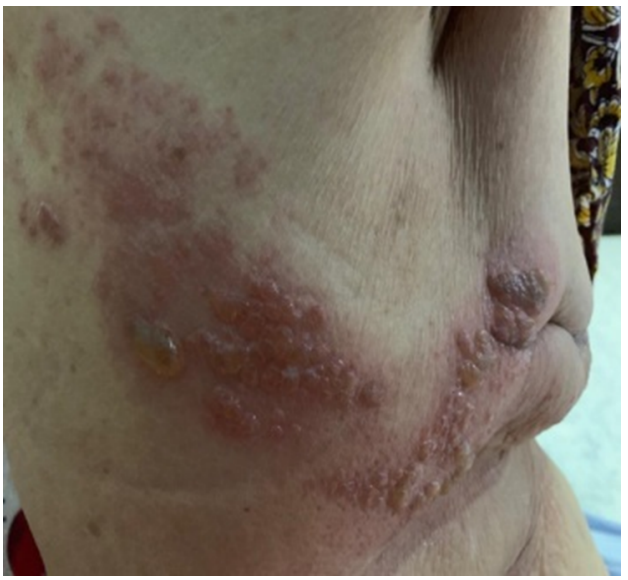

Sheet Of Varicella Zoster Shingles 
REFERENCES:

1. Mishra MV, Keith SW, Shen X, Bar AV, Champ CE, Biswas T. Primary pancreatic lymphoma: a population based analysis using the SEER program. Am J Clin Oncol. 2013; 36(1):38-43[ PubMed].

2. Ramesh J, Hebert-Magee S, Kim H, Trevino J, Varadarajulu S. Frequency of occurrence and characteristics of primary pancreatic lymphoma during endoscopic ultrasound guided fine needle aspiration: $\alpha$ retrospective study. Dig liver Dis.2014; 46(5): 470-473. [ PMC free article] [PubMed].

3. Lin H, Li SD, Hu XG, Li ZS. Primary pancreatic lymphoma: report of six cases. World J Gastroenterol. 2006; 12(31): 5064-5067. [ PMC free article] [PubMed].

4. Dawson IM, Cornes IS, Morson BC. Primary malignant lymphoid tumours of the intestinal tract. Report of 37 cases with a study of factors influencing prognosis. BrJ Surg. 1961; 49: 80-89[PubMed].

5. Saif MW. Primary pancreatic lymphomas. JOP.2006; 7(3): 262-273. [PubMed].

6. Salvatore JR, Cooper B, Shah I, Kummet T. Primary pancreatic lymphoma : a case report, literature review, and proposal for nomenclature. Med Oncol. 2000: 17(3): 237-247 [PubMed].

7. Tuchek JM, De Jong SA, Pickleman J. Disgnosis, surgical intervention, and prognosis of primary pancreatic lymphoma. Am Surg. 1993; 59(8): 513518.[PubMed].

8. Lungu O, Annunziato PW, Gershon A, Staugaitis SM, Josefson D, LaRussa P Silverstein SJ. Reactivated and latent varicella- zoster virus in human dorsal root gangia. Proc Natl Sci USA. 1995; 92: 10980-10984.[ PMC free article] [PubMed].

9. Frossard JL, Steer ML,, Pastor CM. Acute pancreatitis. Lancet.2008; 371: 143152. [PubMed]

10. Stratman E. Visceral zoster as the presenting feature of disseminated herpes zoster. J Am Acad Dermatol. 2002; 46: 771-774. [PubMed]

11. Kawakami T, Mitsui T, Sendo D, Kanazawa C, Maeda S, Katsuura M, Shimuzu Y, Yokoyama S. Acute pancreatitis following hematopoietic stem cell transplantation: prevalence and cause of pancreatic amylasemia. Rinsho Ketsueki. 2002; 43: 176-182.[PubMed].

12. Remmerswaal RG, de Vries AC, Ramsoekh D, van Buuren HR. Varicella zoster- associated gastric ulcers, hepatitis and pancreatitis in an immunocompromised patient. Endoscopy. 2012;44 Suppl 2 UCTN:E 140.[PubMed] 